\title{
Consideration on the effects of DERs in the PLC communication channel
}

\author{
J.-I. Cairo ${ }^{1}$, Jordi Pegueroles, Fernando Martín, Maite Hormigo \\ ${ }^{1}$ IREC, Catalonia Institute for Energy Research \\ C. Jardins de les Dones de Negre, 1, Pl. 2a, 08930 Sant Adriá del Besós, Barcelona, Spain \\ Phone: +34 933562 615. e-mail: icairo@irec.cat
}

\begin{abstract}
In the intend to use the transmission and distribution power lines as a communication channel, much attention has been provided in the modeling of the transmission media used for the propagation of the signals that transmit the information. Most of this effort has been applied in the broadband propagation characteristics, due to higher bandwidth and the use of higher frequencies that can avoid some interference [1]. In this paper it is proposed a method to better understand parameter differences between line characterization in the different frequencies of interest for Power Line Communications (PLC) applicable to analytically determine the channel model, by analyzing the effects of reflections due to the nodes and branch lines impedance mismatches that are present at the distribution power lines. Nowadays such methodology is empirically determined, so this new approach intends to facilitate the modeling methodology prior to its implementation.
\end{abstract}

\section{Key words}

Noise, attenuation, Distributed energy resources, power converters

\section{Introduction}

The active distribution network requires advanced information, communication and control technology to be the basic support that depends on the reliability of data transmission via the information and communication network. In the intend to use the transmission and distribution power lines as a communication channel for the new interconnected active distribution power grid [2], much attention has been provided in the modeling of the transmission media used for the propagation of that signals.

One of the pillars of smart grids is the capability of collecting and managing data from meters in a remote way, using a bidirectional communication link. Smart metering is being deployed all over the world, and power line communications is frequently used to provide this communication over the existing power network, enabling Utilities to implement cost-effective AMI solutions.

PRIME standard is being widely used in Utilities for this purpose since the foundation of the PRIME Alliance, in 2009. It comprehends a non-proprietary, license free open standard, reducing the meter device cost (CAPEX), and the communication carrier cost (OPEX), as it uses powerline communication signals in the 3 to $148.5 \mathrm{kHz}$ CENELEC or the 14 to $480 \mathrm{kHz}$ FCC frequency bands.

Until now little work has been done on modeling low frequency PLC channel [2] - [3], which contrast on the high use for electrical utilities in regards to AMR deployment. So a contribution of this work is made, based not only applying the models for power line channels, or impedance effects of typical appliances [4], or just to the power lines including noise [5], but also on analyzing rigorously the effect of DER units and impedance matching in the whole distribution system.

A method is proposed to analyze line characterization in the different frequencies of interest for PLC to analytically determine the channel model, by analytically analyzing the effects of reflections and impedance mismatching due to the nodes and branch lines impedance mismatches, that are present at the distribution power lines, terminal impedances such as the ones presented by DER, power distribution transformer, and the common terminal impedances presented by home power lines. Nowadays such methodology is partially empirically determined [6] so this new approach intends to facilitate the modeling methodology prior to its implementation. When utilities deploy the NB-PLC system for AMI purposes, it is of much importance to understand the media where the signal will be propagated.

The methodology merges several analytical approaches, making particular emphasis on distributed impedances over distribution grids with impedance effects made by substation power transformer, DER units, and domestic power grids that might affect the PLC signal propagation,.

\section{Power line parameter extraction}

A typical power line cable consists of three conductors; phase, neutral and ground. Each of the neutral and phase are coated by an insulator and the set of three conductors can be surrounded by metal cover or mostly by another insulator. Two of these three conductors are sufficient to create a communication channel.

Transmission lines are characterized by a combination of series and parallel components that represent different physical aspects of the lines. When proposing the modeling of power lines for PLC communication, several 
approaches have been presented using parameter fitting approach [3] based on some measurements and adjusting the values based on such measurements, or the use a bottom-up approach, using physical properties of the cables in order to obtain cable models. In the case here presented, based on physical parameters of the cables, the electrical parameters are extracted. Such parameter extraction is considered representative for other implementations, since must link the real physical implementation with the analytic model.

\section{Use case presentation}

The proposed use case under study, considers the interaction between part of a power distribution grid with local loads $\left(L V_{d i s}\right)$, and a domestic or internal power grid $\left(L V_{d o m}\right)$. The main grid consist of the connection from a substation distribution transformer, taking the feeder output of such transformer down to the end user.

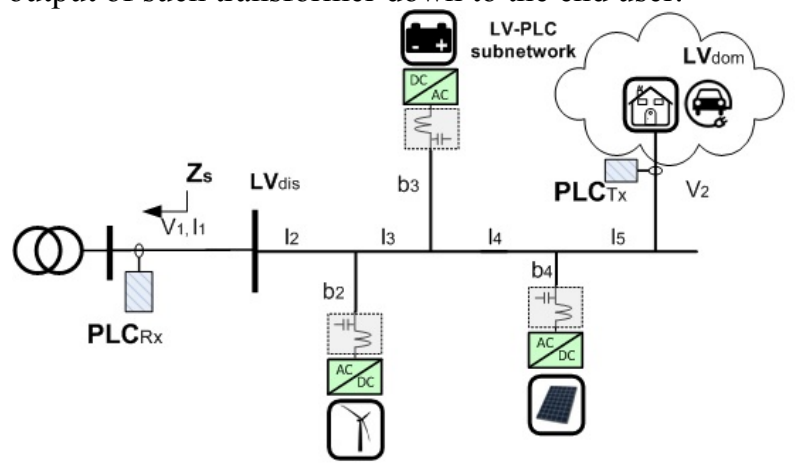

Fig. 1. Power grid with the PLC concentrator and smart meters

Such power grid is characterized by presenting a wide section cable, long distance, with few branch line connections that interface with the loads and the final users. These branch lines are responsible of reflections that will incur in signal attenuation. A residential distribution grid is deployed with some more connections of internal branch lines, presenting much shorter distance and smaller cable section. The loads attached to such power lines present a higher level of impedance unpredictability due to its random nature of use and wide range of possible impedance values varying from few $\Omega$ to $\mathrm{k} \Omega$ [7]. Even with short distances, the losses are very high, due to a dense number of branches and the absence of impedance matching. For certain frequency bands, the fluctuations observed in the channel transfer function within the same mains cycle can reach up to $5 \mathrm{~dB}$

\section{A. Model for the LV distribution grid}

The study performed of the PLC channel transfer function is based on chain-matrix (ABCD matrix) theory [8] very convenient for the calculation of channel transfer functions [9], including intermediate impedances and loads. In particular our distribution system can be implemented by means of modelling the following components represented in figure 2 :

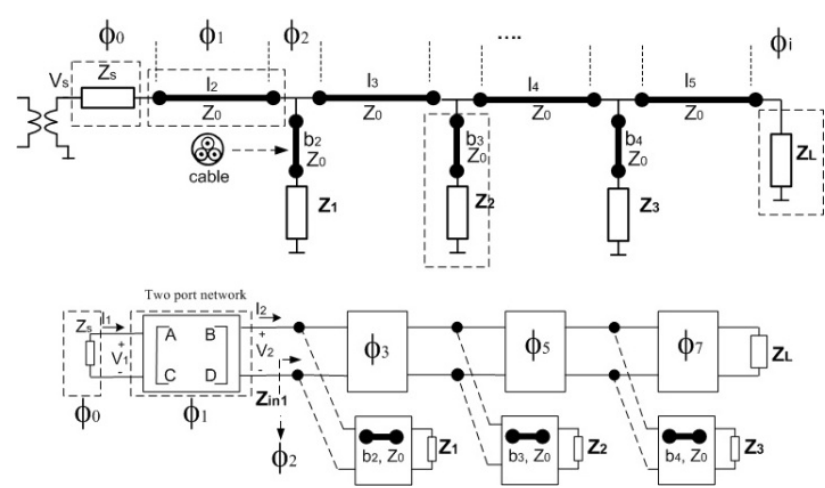

Fig. 2. Equivalent model of the power grid for PLC analysis.

\section{Source impedance}

Expressed in terms of $\mathrm{ABCD}$ matrix in the following way

$$
\emptyset_{0}=\left[\begin{array}{cc}
1 & Z_{s} \\
0 & 1
\end{array}\right]
$$

\section{Parallel transmission line}

Can be implemented by the ABCD matrix defined by $\Phi_{i}$ for the parallel cable with length $l_{i}$, characteristic impedance $Z_{0}$, and propagation constant $\gamma$ :

$$
\begin{gathered}
\emptyset_{1}=\left[\begin{array}{ll}
A & B \\
C & D
\end{array}\right]=\left[\begin{array}{ll}
\cosh \left(\gamma_{i} l_{i}\right) & Z_{c} \sinh \left(\gamma_{i} l_{i}\right) \\
\frac{1}{Z_{c}} \sinh \left(\gamma_{i} l_{i}\right) & \cosh \left(\gamma_{i} l_{i}\right)
\end{array}\right] \\
Z_{0}=\sqrt{\frac{R_{L F}+j \omega L_{L F}}{G+j \omega C}} \\
\gamma_{i}=\sqrt{\left(R_{L F}+j \omega L_{L F}\right)(G+j \omega C)}
\end{gathered}
$$

\section{Bridge tap}

A transmission line with length $b_{i}$, ended with load impedance $Z_{i}$, that are represented by its equivalent input impedance from the main network by:

$$
Z_{e q}=Z_{0} \frac{Z_{i}+Z_{0} \tanh \left(\gamma_{i} b_{i}\right)}{Z_{0}+Z_{i} \tanh \left(\gamma_{i} b_{i}\right)}
$$

\section{Terminal load}

Is represented by the $\mathrm{ABCD}$ matrix expressed in the following manner

$$
\emptyset_{2}=\left[\begin{array}{ll}
1 & 0 \\
\frac{1}{Z_{L}} & 1
\end{array}\right]
$$

The use of the previous matrices will be applied to calculate the transfer function from the circuit, being able to represent the effects of the different loads and wiring from the input or output point of view. This is done by multiplication of the ABCD matrices $\left(\Phi_{T}\right)$, so the relation from PLC-concentrator voltage $\left(V_{l}\right)$ to the voltage at PLCmeter $\left(V_{2}\right)$ is: 


$$
H(f)=\frac{V_{2}}{V_{1}}=\frac{Z_{L}}{A_{\emptyset T} Z_{L}+B_{\emptyset T}+C_{\emptyset T} Z_{S} Z_{L}+D_{\emptyset T} Z_{S}}
$$

\section{B. Model for the $M V / L V$ distribution transformer}

In addition to the model of power lines and loads, in the case presented, it is also necessary to develop a model of the power distribution transformer, responsible of transforming medium voltage (MV) into low voltage (LV), where the PLC concentrator is connected to. In this study the model from [10] is used, and presented in Fig.3. The equivalent input impedance of the transformer for the frequency of interest in the LV side is extracted, having a value of $20 \mathrm{Ohm}$ with a phase of $50^{\circ}(\mathrm{R}=12.8 \Omega, \mathrm{L}=$ $24.3 \mu \mathrm{H})$.

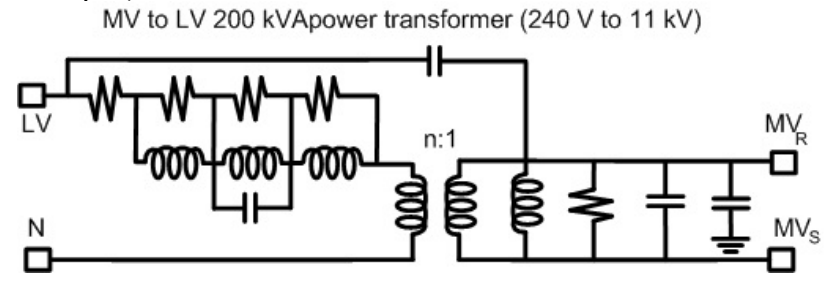

Fig. 3. Equivalent circuit for the MV to LV power transformer.

\section{Model for the DER's impedance}

For the case of DER units, they are measured in a laboratory, in which the power converters define a microgrid environment, where different elements are configured as generation systems (PV or wind), storage (any kind of storage) and loads. In any case they are interface by means of back-to-back power converters with an output filter coil, that present a similar impedance to the network, independently of the power level, absorbed or delivered into the grid.

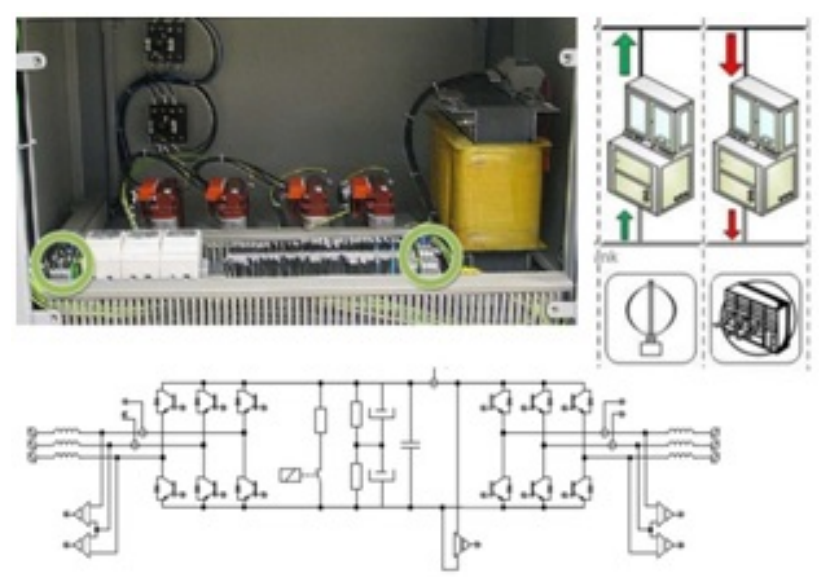

Fig. 4. DER unit with the VSC schematic and output filter coil.

In order to characterize the output filter behaviour, it will be compared the equivalent inductor circuit and the experimental measurement of a real power electronic converter output filter. The resistance of the inductor's coils is considered a parasitic component of the inductor impedance and designated as $R_{p a r}$. The proximity of the adjacent inductor coils introduces a parasitic capacitance component into the inductor equivalent impedance. This parasitic capacitance, designated as $C_{p a r}$, increases significantly when space saving winding techniques (such as multiple layers of coils) are employed. the equivalent model can be expressed as a series combination of the element inductance and the parasitic resistance.

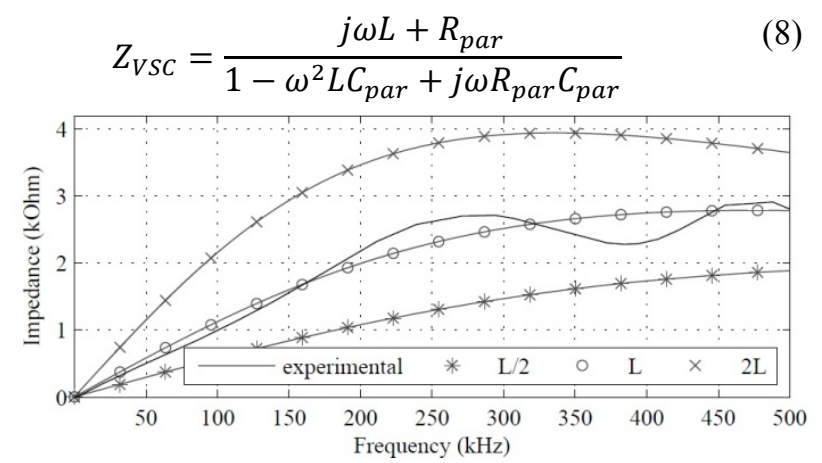

Fig. 5. Measurements of the VSC ouput filter coil.

\section{Simulation results}

Two simulations are conducted, one for the domestic power grid, and another for the distribution grid, using the previous obtained models of impedances from the domestic grid, and also the impedance presented by the distribution power transformer in the substation.

\section{A. Domestic distribution grid}

A domestic $\mathrm{LV}$ power grid is simulated in order to obtain order of magnitude and variability of impedances and attenuation in the same band as the NB-PLC signal. Table I shows grid configuration. For the simulation $A B C D$ matrix method is used. In that case to show variability of the channel, three simulation setups have been considered for the case of having just one, two or three branch line ramifications from the main domestic distribution power line. Attenuation can reach $-35 \mathrm{~dB}$ at $17 \mathrm{MHz}$. The cable in this case is thinner that in distribution grids. We use the same cable permeability $\left(\varepsilon_{r}=4.5\right)$, the main difference resides in having much shorter cable lengths. At this frequency range, the characteristic impedance will not vary as in the NB-PLC case, so will keep constant for the frequency of interest $\left(\left|\mathrm{Z}_{0 \mathrm{~A}}\right|=100 \Omega\right.$ and $\left.\left|\mathrm{Z}_{0 \mathrm{~B}}\right|=110 \Omega\right)$.

TABLE I. NB-PLC channel layout values

\begin{tabular}{lllll}
\hline & T.lines & Line $(\mathrm{m})$ & Branch $(\mathrm{m})$ & Terminal load \\
\hline \hline (a) & 2 & 1,5 & 2 & $\mathrm{Z}_{\mathrm{La}}=50+\mathrm{j} 100$ \\
\hline (b) & 3 & $1,5,7$ & $1.5,2.5$ & $\mathrm{Z}_{\mathrm{Lb}}=150$ \\
\hline (c) & 4 & $1,5,7,5$ & $1.5,2.5,1$ & $\mathrm{Z}_{\mathrm{Lc}}=20-\mathrm{j} 100$ \\
\hline
\end{tabular}

For the prove of consistency with the aim of comparing with other methods, the attenuation is also computed by using the well known Zimmermann method [11-12-13], derived using similar concept as for multipath wireless channels, frequency response imposed to several multipath.

$$
H(f)=\sum_{i=1}^{N} g_{i} e^{\left(-\alpha_{0}+\alpha_{1} f^{k}\right) d_{i}} e^{\left(-j 2 \pi\left(d_{i} / v_{p}\right)\right.}
$$



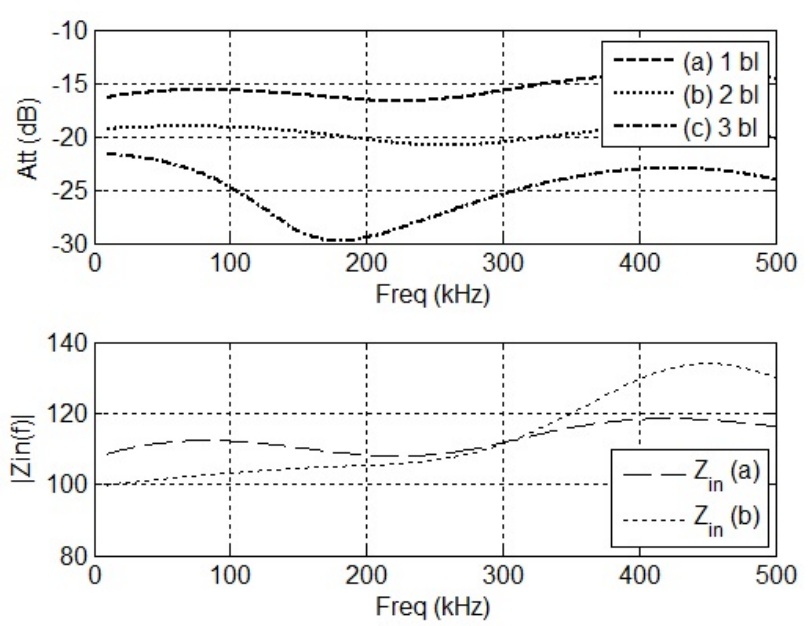

Fig. 7. LV domestic grid (NB-PLC) simulation for set-up as table I. Top: Attenuation for 1 to 3 brach line (bl.). Bottom: Impedances seen at the bl. node.

with $g_{i}$ is the $i_{t h}$ weighting factor from transmission and reflection, $\alpha_{0}$ and $\alpha_{01}$ are the attenuation factor, $d_{i}$ is the length of the $i_{t h}$ path, $v_{p}$ is the speed of light transmitting in the insulating material, parameters to be empirically obtained $\left(, \alpha_{0}=1 \mathrm{e}-3, \alpha_{01}=2.5 \mathrm{e}-9\right.$, obtained from [13].

\section{B. Distribution grid}

The grid under study is described in figure 1. From the power distribution transformer, location where a PLC concentrator is located, until the terminal load where the domestic grid is located and, where a metering system with PLC is plugged, the different models from the elements of the system are modelled according to the parameter values are described in table II.

Attenuations of the channel for the frequencies of interest in NB-PLC are depicted in figure 8. The simulations have been reproduced for different $L V_{\text {dis }}$ configurations, to represent that the attenuation is modified accordingly depending on the number of branches and length of the different wiring segments.

TABLE II. NB-PLC channel layout values (wire 4x95)

\begin{tabular}{lllll}
\hline & T.lines & Line $(\mathrm{m})$ & Branch $(\mathrm{m})$ & Terminal load \\
\hline \hline (a) & 2 & 20,50 & 20 & $\mathrm{Z}_{\mathrm{L} 1}=50+\mathrm{j} 100$ \\
\hline (b) & 3 & 10 & 10 & $\mathrm{Z}_{\mathrm{L} 2}=150$ \\
\hline (c) & 4 & 40 & 30 & $\mathrm{Z}_{\mathrm{L} 3}=20-\mathrm{j} 100$ \\
\hline (d) & 5 & 15 & 10 & $\mathrm{Z}_{\mathrm{L} 4}=20-\mathrm{j} 20$ \\
\hline
\end{tabular}

As a final result, from the methodology presented, one can obtain an idea of the attenuation that the NB-PLC signal would suffer when being transmitted by such media, with different sort of load topologies, from fixed value terminal impendance, to DER units interfaced by back-to-back power inverter and a terminal lof frequency filter, and also the substation transformer, that supplies not only this distribution line, but also other lines with their respective feeders.

In table III, one can see a summary of the order of magnitude for the attenuation, when including such wide range of variability loads.
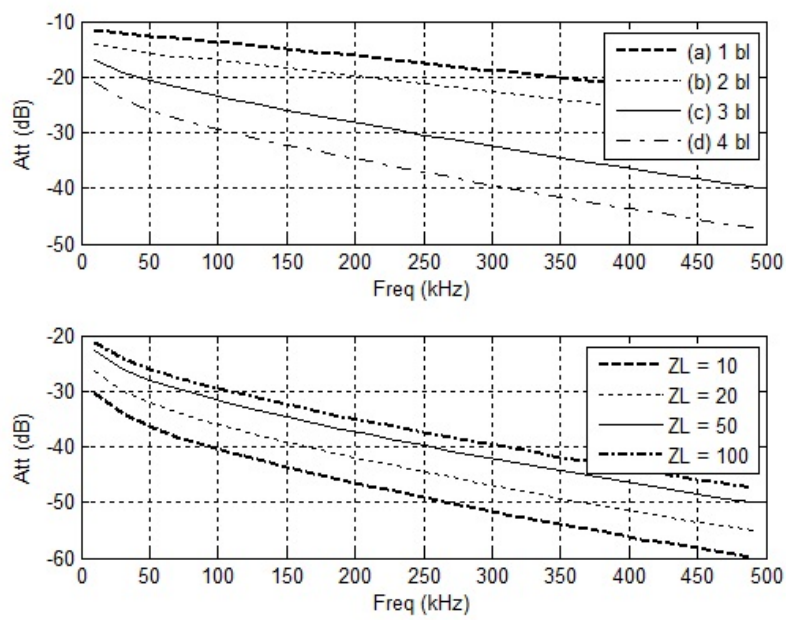

Fig. 8. Attenuation in the distribution grid, based on the set-up presented in table II.

TABLE III. Global simulation results

\begin{tabular}{lll|ll}
\hline & \multicolumn{2}{c|}{ Domestic LV grid } & \multicolumn{2}{c}{ Distribution Grid } \\
\#b.1. & $1_{\mathrm{TOT}}(\mathrm{m})$ & $\operatorname{Att}(\mathrm{dB})$ & $1_{\mathrm{TOT}}(\mathrm{m})$ & $\operatorname{Att}(\mathrm{dB})$ \\
\hline \hline 1 & 60 & 15 & 12 & 12 \\
\hline 2 & 130 & 19 & 15 & 15 \\
\hline 3 & 180 & 25 & 24 & 24 \\
\hline 4 & & & 30 & 30 \\
\hline
\end{tabular}

\section{Conclusions}

The work, shows a methodology that allows to present estimative results on the behaviour of communications signals in the CENELEC frequency band by looking at the variability of the attenuation of the propagation medium, taking as a premise, information on network topology, physical wire characteristics, DER terminal impedances, distribution transformer impedance and domestic grid. In reference to the terminal loads, it has been shown how useful can be the modelling of local domestic grid, and consider just its frequency variation, both terminal impedances as well as characteristic impedance.

The simulation methodology presented could be integrated in a global simulation that by including the noise sources from the different components, would allow an accurate estimation of the modulated signal performance. Such estimation would contribute to obtain some figure of merits characteristics of the telecommunication systems.

\section{Acknowledgement}

The research leading to these results has received funding from the European Union seventh framework program FP7-SMARTCITIES-2013 under grant agreement 608860 in the project IDE4L.

\section{References}

[1] H. Meng, S. Chen, L. Guan, C.L. Law, P.L. So, E. Gunawan, and T. T. Lie, 2002, " A transmission line model for high-frequency power line communication channel ", Int. Conf. on Power System technology, pp. 1290-1295, 
[2] A. Pinomaa, J. Ahola, and A. Kosonen, 2012, "Channel model for a Power Line Communications medium in an LVDC distribution system", IEEE Int'l Symp. on Power Line Communications and its Applications, pp. 404-410.

[3] A. M. Tonelllo, F. Versolatto, B. Béjar, and S. Zazo, 2012," A fitting algorithm for random modeling the PLC channel ", IEEE Trans. on power delivery. pp. 1477-1484.

[4] A.Achouri, J-C. Lebunetel, Y. Raingeaud, R.Nizigiyimana, "Impedance impact on indoor narrowband power line channel", Proc. Of the 2014 International Symposium on Electromagnetic Compatibility (EMC Europe 2014),pp.1155-1160.

[5] J. Matanza, S. Alexandres, C.Rodríguez-Morzillo, "Advanced metering infrastructure performance using European low-voltage power line communication networks", IET Communications, Sept. 2013, p.1041-1047.

[6] G. Chu, J.Li, W.Liu, "Narrow band power line cannel characteristics for low voltaje Access network in China", 2013 IEEE 17th International Symposium on power line communications and its applications, pp.297-302.

[7] F.J. Canete, J.A. Cortés. L. Diez, J.T. Entrambasaguas," Modeling and evaluation of the indoor Power Line Transmission Medium", IEEE Comm. Magazine, 2003

[8] G. Temes, J. Lapatra, "Introduction to Circuit Synthesis and Design" McGraw-Hill: N.Y., 1967

[9] J. Irwin, "Basic Engineering Circuit Analysis" MacMillan: New York, 1987.

[10] C.J.Kikkert, 2012, "A PLC Frequency Model of 3 Phase Power Distribution Transformers ", IEEE SmartGridComm 2012 Symposium - Communication Networks for Smart Grids and Smart Metering, 205-210

[11] M. Zimmermann, and K. Doster,2002, “A multipath model for the power line channel", IEEE Trans. on Communications, vol.50, pp.553-339.

[12] O.G. Hooijen,2002, "A channel model for the residential power circuit used as a digital communications medium", IEEE Transactions on ELectromagnetic Compatibility, vol. 44,

[13] IEEE Std 1901.2, "Low-Frequency (less than 500 $\mathrm{kHz}$ ) Narrowband Power Line Communications for Smart Grid Applications", IEEE Communications Society, 2013 